\title{
Efficience of Cardiopulmonary Resuscitation in Emergency Medical Service and University Hospital
}

\author{
Anita Kaleja*, Elina Snucina**, Indulis Vanags* \\ *Pauls Stradins Clinical University Hospital, Department of Anaesthesiology and Reanimatology, Riga, Latvia \\ **Riga Stradins University, Faculty of Continuing Education, Latvia
}

\begin{abstract}
Summary
Introduction. Survival rate among patients with cardiac arrest remains unclear. Return of spontaneous circulation (ROSC) is just the first step toward the goal of complete recovery from cardiac arrest. It depends on quality of cardiopulmonary resuscitation (CPR) during resuscitation and factors of postresuscitation care. Regular CPR analysis has not been carried out in Latvia.

Aim of the study was to evaluate survival rate among patients with out-of-hospital cardiac arrest and in-hospital cardiac arrest.

Materials and methods. The study was conducted in the State Emergency service of Latvia and Pauls Stradins Clinical University Hospital during 15 months in 2010/2011. There were 221 adult patients with in-hospital cardiac arrest and 162 adult patients with out-of-hospital cardiac arrest and performed CPR included in retrospective research. The information was analyzed by medical records. The obtained results were expressed in percents and compared, using the Pearson's Chi-square (Pearson X2) test.

Results. The short-term ROSC was achieved among patients with out-of-hospital cardiac arrest (OHCA) in 62 cases and among patients with in-hospital cardiac arrest (IHCA) in 186 cases. Survival to discharge was achieved in $20.3 \%$ among patients with $0 \mathrm{HCA}$ and $15.8 \%$ among patients with IHCA. The most commonly used CPR algorithm was pulseless electrical activity/asystole (72 - 73\%). Short-term ROSC was achieved most frequently by ventricular fibrillation/pulseless ventricular tachycardia (41.3-56\%), but the largest number of unsuccessful CPR episodes was observed by pulseless electrical activity/ asystole.
\end{abstract}

Conclusion. Results of CPR were different among patients with OHCA and IHCA. ROSC is rhythm-specific outcome.

Key words: in-hospital cardiac arrest; out-of-hospital cardiac arrest; return of spontaneous circulation; CPR.

\section{INTRODUCTION}

Cardiopulmonary resuscitation (CPR) is a complex of emergency procedures that, if performed correctly, can provide the necessary minimum of circulation until the return of spontaneous circulation (ROSC) is achieved $(3 ; 4)$. ROSC is just the first step toward the goal of complete recovery from cardiac arrest. It depends on quality of CPR during acute resuscitation and factors of postresuscitation care. Survival rate among patients with cardiac arrest remains unclear. There is high heterogenity among CPR studies and they are not comparable (12). The European Resuscitation Council (ERC) Guidelines 2010 emphasize the quality of cardiopulmonary resuscitation and the postresuscitation care as the big challenge for the next five years (5). Despite new techniques and technology it is not clear whether survival after cardiac arrest have improved (10). Therefore it is important to analyze the factors, determining the efficiency of the CPR such as patient's location at the moment of cardiac arrest: outof-hospital or in-hospital $(1 ; 11)$, the pathogenetic mechanism of the cardiac arrest: ventricular fibrillation/ pulseless ventricular tachycardia, pulseless electrical activity, asystole $(2 ; 8 ; 9)$, implementation or improvement of training for medical persons, implementing of the resuscitation protocols in hospitals, the quality control of CPR during performing (4). The most common survival rate to hospital discharge range is reported being between $13.7 \%$ and $22.3 \%$ (10). It is worth mentioning that in the majority of cases it is possible to assess the quality of CPR only indirectly, according to the manikin studies in the process of training or according to the experimentally obtained data. The efficiency of CPR in decreasing the deathrate from avertable causes is a very important factor. In Latvia such systematic studies are not conducted at all (6). Therefore we should analyze the results and efficiency of CPR both out-of-hospital and in-hospital and look for ways to improve the situation in the future (3). The results of cardiopulmonary resuscitation during out-of-hospital cardiac arrest and in-hospital cardiac arrest were evaluated in that research - OHCA versus IHCA.

\section{AIM OF THE STUDY}

The aim of our study was to evaluate survival rate among patients with out-of-hospital and in-hospital cardiac arrest.

\section{MATERIALS AND METHODS}

The study was conducted in State Emergency Medical Service of the Republic of Latvia and in the Pauls Stradins Clinical University Hospital during 15 months in 2010/2011. Survival outcomes were evaluated using prehospital and hospital medical records. Cardiopulmonary resuscitation was performed according to the ERC Guidelines 2005 and 2010. There were 687 adult patients with a confirmed cardiac arrest, defined as unresponsiveness, apnea and the lack of 
signs of circulation and performed CPR included in the retrospective research. 451 patient records with OHCA and 236 patient records with IHCA were analyzed. There were following factors evaluated: cardiac arrest location; medical team, who provided CPR; the pathogenetic mechanism of the cardiac arrest (ventricular fibrillation/ pulseless ventricular tachycardia, pulseless electrical activity, asystole) and survival outcomes. It was considered that CPR is effective if return of spontaneous circulation was established. Survival outcomes were categorized as short-term ROSC and long-term ROSC (7; 12). A short-term ROSC was defined as the return of spontaneous circulation for at least 20 minutes after the initial pulseless arrest and survival to hospital admission. A long-term ROSC was defined as hospital discharge. 304 patients were excluded from study there were trauma patients, oncological patients, who received palliative care, and cases with missing data. Patients with OHCA were excluded from IHCA study. All CPR episodes were included for IHCA patients with multiple cardiac arrests (Table 2). The further analysis was conducted on 162 OHCA patients and 221 IHCA patients.

All OHCA occurred in presence of emergency medical service team. IHCA pacients were treated in general intensive care unit (ICU) and cardiological ICU with monitoring and medical staff on duty.

It was established that emergency medical service team and medical staff in hospital were similarly equipped with monitors, providing continuous registration of the vitally important parameters: the heart rhythm and heart frequency, the non-invasive arterial blood pressure and the level of oxygen saturation in blood. CPR was performed by trained prehospital personnel such as emergency physicians/reanimatologists, doctors assistants and trained ambulance car drivers and by hospital personnel such as physicians (emergency physicians/reanimatologists or cardiologists and trained nurses). An indication for admitting patients in the intensive care setting was haemodinamically unstable condition, a necessity for intensive treatment and the risk of cardiac arrest from reversible causes.

The impact of pathogenetic mechanism of cardiac arrest were analyzed in both groups, including ventricular fibrillation/ pulseless ventricular tachycardia - VF/VT, the pulseless electrical activity/ asystole - PEA/A and indeterminate rhythm.

Survival to hospital admission, survival to discharge and rhythm-specific survival was assessed in OHCA study. Short-term survival, survival to discharge and rhythm-specific survival were assessed in IHCA study. The obtained results were expressed in percents and compared, using the Pearson's Chi-square (Pearson $\chi^{2}$ ) test. The statistically valid $p$ value was $<0,05$.

\section{RESULTS}

Among included pacients mean age for male was 64 years, for female 70.5 years; most of patients were male (Table 1). The most frequent basic disease in IHCA study (Table 1$)$ was of cardiac origin $(n=101 ; 46 \%)$, folloved by diseases of neurological origin $(n=51 ; 23 \%)$. The baseline diagnosis in OHCA study was identified in $76.9 \%$ only, data were incomplete. Cardiac origin was assumed in $78 \%$ potencially identified OHCA diagnosis. There were 221 adult patients and 252 CPR episodes included in the IHCA study, the cardiopulmonary resuscitation on 31 patients was performed more than one episode.

The obtained data in IHCA study (Table 1) shoved that pulseless electrical activity/ asystole (PEA/A) was the initial heart rhythm in $73 \%$, ventricular fibrillation/ pulseless ventricular tachycardia (VF/VT) in $23 \%$ and the initial heart rhythm was not determinate in $4 \%$. ROSC was achieved most frequently in ventricular fibrillation/ pulseless ventricular tachycardia (Table 3), but unsuccessful CPR episodes were more common in pulseless electrical activities/ asystole. Using the parametric Pearson's chi-square (Pearson $\chi^{2}$ ) test, the value $\mathrm{p}$ was obtained, which indicated that the rhythmspecific differences of ROSC frequency were statistically valid (Chi2 $\mathrm{p}<0.0001$ ).

OHCA data showed that initial heart rhythm was nonshockable (PEA/A) in $72.1 \%$ and shockable rhythm(VF/ pulseless VT) was present in $27.9 \%$ (Table 1). The rhythm - specific short-term ROSC was achieved most frequently in cases of VF/pulseless VT (Table 3) and the difference was statistically valid $(\mathrm{p}<001)$.

Short-term ROSC was achieved in IHCA study (Table 2 ) frequently $(n=186 ; 84,2 \%)$, but the number of patients who were dischargexd from the hospitals after cardiac arrest was small $(n=35 ; 15,8 \%) .62$ patients in OHCA study (Table 2) were admitted to Pauls Stradins University Hospital and Riga Eastern University Hospital after cardiac arrest and 33 patients (20.3\%) were discharged alive.

\section{DISCUSSION}

CPR is one of the most important emergency actions in life-threatining conditions. Wallace et al. showed high heterogenity among studies related to the efficiency of CPR, suggesting that they are not comparable (12). The aim of this study was to assess the results and their determining factors of IHCA and OHCA, such as the efficiency of CPR depending on the patients location, and various pathogenetic mechanisms of the cardiac arrest. The demographic characteristics of patients age group corresponded with the general demographic trends. The average age between the genders did not differ much - it was 70.5 years for females, 64 years for males, however most of patients were male. The most common cause of cardiac arrest was on cardiac origin, which also corresponds with the statistic data of the Republic of Latvia.

Short-term ROSC was achieved in 186 episodes from 221 patients with IHCA, and 35 patients $(15,8 \%)$ were discharged alive from the hospital. Short-term ROSC was achieved for 62 patients from 162 in OHCA group and $33(20.3 \%)$ were discharged alive. These figures can be compared with the data published in separate studies. Girotra S.et al. shoved in a large, prospective, hospi- 
tal - based, clinical registry of patients with in-hospital cardiac arrests in the United States that the overall rate of survival to discharge was $17 \%$ and there was a significant trend toward increased survival during 2000-2009 (10). The difference between IHCA and OHCA in our study should be evaluated with caution, because confounding factors were not identified and the possibility of residual confounding still remains. Unfortunately there was no accurate information on the neurological outcome in medical records at the time of discharge. Some studies showed that rates of severe neurological disability did not change significantly over time and improvement in survival rate has been accompanied by a decrease in the rate of neurologic disability among survivors (7). There were no detailed information on post cardiac arrest syndrome variables, like therapeutic hypothermia (10). Therapeutic hypothermia remains poorly implemented and further research is required, while the cognitive function is susceptible to many physiological and pharmacological perturbations during the acute periode (7). Very likely that better outcomes can be achieved with cooling but this hypothesis has been proven in clinical studies.

Analyzing the ROSC frequency depending on the pathogenetic mechanism of the cardiac arrest it is evident that the best results can be achieved if the first rhythm was the ventricular fibrillation or pulseless ventricular tachycardia, which corresponds with the data available in the literature. However Girotra S. et al. showed (10) that the proportion of cardiac arrests due to asystole or pulseless electrical activity increased during $2000-2009$ from $68.7 \%$ in 2000 to $82.4 \%$ in 2009 ( $\mathrm{p}<0.001$ for trend)

The following problems were established during the study: 1) selection of primary outcomes varies in both studies; 2) improvements are necessary in documentation of resuscitation efforts, but same factors are often difficult to document accurately; 2) the neurological status were not evaluated. Future studies are needed to understand which factors are responsible for improvements in survival after cardiac arrest.

\section{CONCLUSIONS}

Results of CPR were different among patients with OHCA and IHCA. It should be interpreted in light of potential confounding factors and future studies are needed. We found that return of spontaneous circulation and survival rate are rhythm-specific outcomes. There is a statistically valid difference among ROSC frequence due to shockable and non-shockable rhythms. The ventricular fibrillation/pulseless ventricular tachycardia are associated with higher survival rate.

\section{Conflict of interest: None}

\section{REFERENCES}

1. Abella BS MD, MPhil; Jason P. Alvarado, BA; Helge Myklebust, BEng; Dana P. Edelson, MD; Anne Barry, RN, MBA; Nicholas O'Hearn, RN, MSN; Terry L. Vanden Hoek, MD; Lance B. Becker, MD.
Quality of Cardiopulmonary Resuscitation During In-Hospital Cardiac Arrest // JAMA, January 19, 2005-Vol 293, No. 3

2. Agarwal DA, Hess EP, Atkinson EJ, White RD. Ventricular fibrillation in Rochester, Minnesota: experience over 18 years // Resuscitation 2009;80:1253-8

3. Cooper JA, Joel D. Cooper and Joshua M. Cooper. Cardiopulmonary Resuscitation: History, Current Practice, and Future Direction // Circulation, 2006, 1 14;2839-2849

4. Dana P. Edelson, MD, MS; Barbara Litzinger, BS; Vineet Arora, MD, MAPP; Deborah Walsh, MS, RN; Salem Kim, BA; Diane S. Lauderdale, PhD; Terry L. Vanden Hoek, MD; Lance B. Becker, MD, FAHA; Benjamin S. Abella, MD, MPhil. Improving inhospital cardiac arrest process and outcomes with performance debriefing // Arch Intern Med., 2008, 168(10):1063 - 1069

5. Jerry P.Nolan, Jasmeet Soar, David A.Zideman, Dominique Biarent, Leo Bossaert, Charles Deakin, Rudolph W.Koster, Jonathan Wyllie, Bernd Bottiger European Resuscitation Council Guidelines for Resuscitation 2010//Resuscitation, 2010, 81:12191221

6. Kaleja A., Mikijanska D., Vanags I. Results of Cardiopulmonary Resuscitation during in-Hospital Cardiac Arrest//Acta Chirurgica Latviensis, 2011, (11/2):84-87

7. Lance B.Becker, Tom P.Aufderheide, Romergryko G.Geocadin, Clifton W.Callaway, Ronald M.Lazar, Michael W.Donnino, Vinay M.Nadkarni, Benjamin S.Abella, Christophe Adrie, Robert A.Berg, Raina M.Merchant, Robert E.O'Connor, David O.Meltzer, Margo B.Holm, William T.Longstreth and Henry R.Halperin.Primary Outcomes for Resuscitation Science Studies: A Consensus Statement From the American Heart Association // Circulation, 2011 , 124:2158-2177

8. Meaney PA, Nadkarni VM, Kern KB, Indik JH, Halperin HR, Berg RA. Rhythms and outcomes of adult in-hospital cardiac arrest // Crit Care Med, 2010; 38:101-8

9. Peberdy MA, Ornato JP, Reynolds P, Weik MH. The first documented cardiac arrest rhythm in patients with heart failure // Resuscitation, 2009, $80(12): 1346-1350$

10. Saket Girotra, M.D., Brahmajee K. Nallamothu, M.D., P.H., John A.Spertus, M.D., M.P.H., Yan Li, PH.D., Harlan M.Krumholz, M.D. and Paul S.Chan, M.D. Trends in Survival after In-Hospital Cardiac Arrest// N Engl J Med 2012; 367:1912-20

11. Sandroni C, Nolan J, Cavallaro F, Antonelli M. Inhospital cardiac arrest: incidence, prognosis and possible measures to improve survival //Intensive Care Med, 2007; 33:237-45

12. Sarah K.Wallace, Benjamin S.Abella and Lance B.Becker Quantifying the Effect of Cardiopulmonary Resuscitation Quality on Cardiac Arrest Outcome: A Systematic Review and MetaAnalysis // Circulation, 2013;6;148-156 
Table 1. Baseline characteristics of patients with OHCA and IHCA

\begin{tabular}{|c|c|c|c|}
\hline & & OHCA & IHCA \\
\hline \multicolumn{4}{|c|}{ Demographic characteristics } \\
\hline \multirow{2}{*}{$\begin{array}{l}\text { Age (mean; } \\
\text { years) }\end{array}$} & Female & 73 & 68 \\
\hline & Male & 63 & 65 \\
\hline \multicolumn{2}{|c|}{ Male sex $(\%)$} & 58.5 & 52.0 \\
\hline \multicolumn{4}{|c|}{ Characteristics of cardiac arrest ( $\%)$} \\
\hline \multicolumn{2}{|l|}{ VF } & 26.6 & \multirow[t]{2}{*}{23} \\
\hline \multicolumn{2}{|c|}{ VT (pulseless) } & 1.3 & \\
\hline \multicolumn{2}{|l|}{ PEA } & 52.8 & \multirow[t]{2}{*}{73} \\
\hline \multicolumn{2}{|l|}{ Asystole } & 19.3 & \\
\hline \multicolumn{2}{|c|}{ Not determinate rhythm } & & 4 \\
\hline \multicolumn{4}{|c|}{ Baseline diagnosis (\%) } \\
\hline \multicolumn{2}{|c|}{ Cardiac origin } & 78.6 & 46 \\
\hline \multicolumn{2}{|c|}{ Neurological origin } & & 23 \\
\hline \multicolumn{2}{|l|}{ Others } & 22.4 & 31 \\
\hline
\end{tabular}

\section{Address:}

Anita Kaleja

Department of Anaesthesiology and Reanimatology

Pauls Stradins Clinical University Hospital

13 Pilsonu street

Riga, Latvia, LV-1002

E-mail: anita.kaleja@stradini.lv
Table 2. CPR results for patients with out-ofhospital cardiac arrest and in-hospital cardiac arrest

\begin{tabular}{|l|l|l|}
\hline & OHCA & IHCA \\
\hline Number of patients & 162 & 221 \\
\hline Short-term ROSC & 62 patients & 186 CPR episodes \\
\hline Long-term ROSC & $\begin{array}{l}33 \text { patients } \\
(20.3 \%)\end{array}$ & $\begin{array}{l}35 \text { patients } \\
(15.8 \%)\end{array}$ \\
\hline
\end{tabular}

Table 3. Rhythm - specific return of spontaneous circulation

\begin{tabular}{|c|c|c|c|c|}
\hline & \multicolumn{2}{|c|}{$\begin{array}{l}\text { Short-term } \\
\text { ROSC achieved } \\
(\%)\end{array}$} & \multicolumn{2}{|c|}{$\begin{array}{l}\text { Short-term } \\
\text { ROSC not } \\
\text { achieved } \\
(\%)\end{array}$} \\
\hline & IHCA & OHCA & IHCA & OHCA \\
\hline $\begin{array}{l}\text { VF/VT } \\
\text { pulseless }\end{array}$ & 56 & 41.3 & 44 & 58.7 \\
\hline PEA & \multirow[t]{2}{*}{39} & 15.5 & \multirow[t]{2}{*}{61} & 84.5 \\
\hline Asystole & & 14.9 & & 85.1 \\
\hline
\end{tabular}

